\title{
THE TORSION AND STRETCHING OF SPIRAL RODS (II)*
}

BY H. OKUBO (Tōhoku L'niversity, Sendai, Japan)

The torsion and stretching problems of spiral rods were discussed in a preceding pape:.' There, the equations of equilibrium were expressed in terms of displacements that were independent of the position of the section perpendicular to the axis of a spiral rod. The differential equations for the displacements were integrated for the particular case where the helix angle was small, and the corresponding displacements and stresses were obtained. In the calculations, however, the displacements were preliminarily assumed in special forms, and consequently solution was valid for some special problems. In the previous paper, the displacements for the stretching problem were assumed in forms that reduce to those for a uniform tension in the limit case when the helix angle approaches zero. But when a spiral rod with axis that does not pass the centroid of the cross section, is pulled axially, the displacements must be in forms that reduce to those for a uniform tension combined with a uniform bending moment in the limit case when the helix angle approaches zero. Hence, the validity of the previous solution was restricted to the problem for a spiral rod with axis through the centroid of the cross section.

As in the preceding paper, we take the axis of helix as the axis of $z$, and denote the displacements in $x^{\prime}, y^{\prime}, z$ directions by $u^{\prime}, v^{\prime}$ and $w$ respectively, in which $x^{\prime}$ and $y^{\prime}$ are the axes perpendicular to each other and fixed to a section of the rod perpendicular to $z$. We take for the displacements the expressions

$$
\left.\begin{array}{l}
u^{\prime}=u_{1}-\gamma x^{\prime}-\frac{\gamma^{\prime}}{2}\left(x^{\prime 2}-y^{\prime 2}\right)-\alpha y^{\prime} z+\frac{\beta^{\prime}}{k^{2}}(1-\cos k z-k z \sin k z), \\
v^{\prime}=v_{1}-\gamma y^{\prime}-\gamma^{\prime} x^{\prime} y^{\prime}+\alpha x^{\prime} z-\frac{\beta^{\prime}}{k^{2}}(\sin k z-k z \cos k z), \\
w=w_{1}+\frac{\beta^{\prime}}{k}\left(x^{\prime} \sin k z-y^{\prime} \cos k z+y^{\prime}\right)+\beta z,
\end{array}\right\}
$$

where $u_{1}, v_{1}, w_{1}$ are the functions of $x^{\prime}, y^{\prime}$ and are independent of $z, k$ is a constant which specifies the helix angle, $\alpha, \beta, \beta^{\prime}$ are arbitrary constants, and

$$
\gamma=\frac{1}{2}(1-p) \beta, \quad \gamma^{\prime}=\frac{1}{2}(1-p) \beta^{\prime}, \quad p=\frac{\mu}{\lambda+\mu} .
$$

From (1) we have the cubical dilatation

$$
\Delta=\frac{\partial u_{1}}{\partial x^{\prime}}+\frac{\partial v_{1}}{\partial y^{\prime}}-k D_{2}\left(w_{1}\right)+p \beta^{\prime} x^{\prime}+p \beta,
$$

where

$$
D_{2}=y^{\prime} \frac{\partial}{\partial x^{\prime}}-x^{\prime} \frac{\partial}{\partial y^{\prime}}
$$

*Received January 13, 1953.

${ }^{1}$ H. Ōkubo, Q. Appl. Math. 9, 263-272 (1951). 
The equations of equilibrium for this case can be expressed in the forms

$$
\left.\begin{array}{r}
\frac{\partial \Delta}{\partial x^{\prime}}+p\left\{\nabla_{1}^{2} u_{1}+k^{2} D_{1}\left(u_{1}\right)-2 k^{2} D_{2}\left(v_{1}\right)+\frac{1}{2} k^{2} \gamma^{\prime}\left(x^{\prime 2}-y^{\prime 2}\right)-\beta^{\prime}\right\}=0 \\
\frac{\partial \Delta}{\partial y^{\prime}}+p\left\{\nabla_{1}^{2} v_{1}+k^{2} D_{1}\left(v_{1}\right)+2 k^{2} D_{2}\left(u_{1}\right)+k^{2} \gamma^{\prime} x^{\prime} y^{\prime}\right\}=0 \\
k D_{2}(\Delta)-p\left\{\nabla_{1}^{2} w_{1}+k^{2} D_{1}\left(w_{1}\right)+k^{2} w_{1}-k \beta^{\prime} y^{\prime}\right\}=0,
\end{array}\right\}
$$

where

$$
\nabla_{1}^{2}=\frac{\partial^{2}}{\partial x^{\prime 2}}+\frac{\partial^{2}}{\partial y^{\prime 2}}, \quad \text { and } \quad D_{1}+1=\left(y^{\prime} \frac{\partial}{\partial x^{\prime}}-x^{\prime} \frac{\partial}{\partial y^{\prime}}\right)\left(y^{\prime} \frac{\partial}{\partial x^{\prime}}-x^{\prime} \frac{\partial}{\partial y^{\prime}}\right) .
$$

The differential equations (2) are independent of $z$. Solving the simultaneous equations for $u_{1}, v_{1}$ and $w_{1}$, we can find the displacements from (1). The displacements for a straight rod are readily obtained from (1), by taking the limit case when $k$ approaches zero; thus

$$
\begin{aligned}
& u=-\gamma x-\alpha y z-\frac{\gamma^{\prime}}{2}\left(x^{2}-y^{2}\right)-\frac{\beta^{\prime}}{2} z^{2}, \\
& v=-\gamma y+\alpha x z-\gamma^{\prime} x y, \\
& w=w_{1}+\beta z+\beta^{\prime} x z,
\end{aligned}
$$

where $u_{1}, v_{1}$ are assumed to vanish when $k$ approaches zero.

Assume that $w_{1}$ and $\alpha$ in (3) also vanish when $k$ approaches zero; the corresponding stresses become

$$
X_{x}=Y_{\nu}=X_{\nu}=X_{z}=Y_{z}=0, \quad Z_{z}=\mu(3-p)\left(\beta+\beta^{\prime} x\right) .
$$

This is the solution for a straight rod submitted to simple tension combined with a uniform bending moment. If we assume that $\beta$ and $\beta^{\prime}$, instead of $w_{1}$ and $\alpha$, vanish when $k$ approaches zero, the corresponding stresses become

$$
X_{x}=Y_{\nu}=Z_{z}=X_{\nu}=0, \quad X_{z}=\mu\left(\frac{\partial w_{1}}{\partial x}-\alpha y\right), \quad Y_{z}=\mu\left(\frac{\partial w_{1}}{\partial y}+\alpha x\right) .
$$

This is the solution for the torsion problem of a straight rod.

Consider now a spiral rod of small $k$, pulled by a pair of axial forces. Assuming that $\alpha, w_{1}$ are small quantities of the order $k$ and $u_{1}, v_{1}$ are of the order $k^{2}$, and neglecting the small quanties of the higher order, the equations of equilibrium (2) can be written as follows:

Let us take for $w_{1}$ the expression

$$
\left.\begin{array}{rl}
\frac{\partial \Delta}{\partial x^{\prime}}+p \nabla_{1}^{2} u_{1}+\frac{1}{2} p k^{2} \gamma^{\prime}\left(x^{\prime 2}-y^{\prime 2}\right)-p \beta^{\prime} & =0, \\
\frac{\partial \Delta}{\partial y^{\prime}}+p \nabla_{1}^{2} v_{1}+p k^{2} \gamma^{\prime} x^{\prime} y^{\prime} & =0 \\
\nabla_{1}^{2} w_{1}-2 k \beta^{\prime} y^{\prime} & =0 .
\end{array}\right\}
$$

$$
w_{1}=i\left(f_{3}-\bar{f}_{3}\right)+\frac{1}{3} k \beta^{\prime} y^{\prime 3}
$$


where $f_{3}$ is an arbitrary function, $\bar{f}_{3}$ is a function conjugate with $f_{3}$, and

$$
f_{3}=f_{3}(\zeta), \quad \bar{f}_{3}=\bar{f}_{3}(\bar{\zeta}), \quad \zeta=x^{\prime}+i y^{\prime}, \quad \bar{\zeta}=x^{\prime}-i y^{\prime} .
$$

The displacement $w_{1}$ satisfies the third Eq. (6). Substituting this expression of $w_{1}$ into the first and second equations (6), the equations for $u_{1}$ and $v_{1}$ become

$$
\begin{aligned}
& (1+p) \frac{\partial^{2} u_{1}}{\partial x^{\prime 2}}+\frac{\partial^{2} v_{1}}{\partial x^{\prime} \partial y^{\prime}}+p \frac{\partial^{2} u_{1}}{\partial y^{\prime 2}} \\
& =k\left(f_{3}^{\prime}+\bar{f}_{3}^{\prime}+\zeta f_{3}^{\prime \prime}+\overline{\left.\zeta f_{3}^{\prime \prime}\right)}-\frac{1}{2} p k^{2} \gamma^{\prime} x^{\prime 2}-\frac{1}{4}\left(4-p+p^{2}\right) k^{2} \beta^{\prime} y^{\prime 2},\right. \\
& p \frac{\partial^{2} v_{1}}{\partial x^{\prime 2}}+\frac{\partial^{2} u_{1}}{\partial x^{\prime} \partial y^{\prime}}+(1+p) \frac{\partial^{2} v_{1}}{\partial y^{\prime 2}} \\
& =i k\left(f_{3}^{\prime}-\overline{f_{3}^{\prime}}+\zeta f_{3}^{\prime \prime}-\overline{\left.\zeta f_{3}^{\prime \prime}\right)}-\frac{1}{2}\left(4+p-p^{2}\right) k^{2} \beta^{\prime} x^{\prime} y^{\prime} .\right.
\end{aligned}
$$

Integrating the differential equations (8), we find

$$
\left.\begin{array}{rl}
u_{1}=f_{1} & +\bar{f}_{1}+x^{\prime}\left(f_{2}+\bar{f}_{2}\right)+k \int f_{3}^{\prime} \zeta d \zeta+k \int \bar{f}_{3}^{\prime} \bar{\zeta} d \bar{\zeta} \\
& +\frac{k^{2} \beta^{\prime}}{48}\left\{p(3-p) x^{\prime 4}-6\left(4+p-p^{2}\right) x^{\prime 2}{y^{\prime 2}}^{2}+\left(6-p-p^{2}\right) y^{\prime 4}\right\}, \\
v_{1}=i\left(f_{1}-\bar{f}_{1}\right)+i x^{\prime}\left(f_{2}-\bar{f}_{2}\right)+i(1+2 p)\left(\int f_{2} d \zeta-\int \bar{f}_{2} d \bar{\zeta}\right),
\end{array}\right\}
$$

where $f_{1}, f_{2}$ are arbitrary functions of $\zeta$. The corresponding stresses become

$$
\begin{aligned}
& X_{z^{\prime}}^{\prime}=2 \mu\left\{f_{1}^{\prime}+\bar{f}_{1}^{\prime}+x^{\prime}\left(f_{2}^{\prime}+\bar{f}_{2}^{\prime}\right)+p\left(f_{2}+\bar{f}_{2}\right)+k\left(\zeta f_{3}^{\prime}+\overline{\zeta f}_{3}^{\prime}\right)\right. \\
& \left.+\frac{1}{24}(3-p)(1+p) k^{2} \beta^{\prime} x^{\prime 3}-\frac{1}{8}\left(9-p^{2}\right) k^{2} \beta^{\prime} x^{\prime} y^{\prime 2}\right\} \\
& Y_{y^{\prime}}^{\prime}=-2 \mu\left\{f_{1}^{\prime}+\bar{f}_{1}^{\prime}+x^{\prime}\left(f_{2}^{\prime}+\bar{f}_{2}^{\prime}\right)+(2+p)\left(f_{2}+\bar{f}_{2}\right)\right. \\
& \left.-\frac{1}{24}(3-p)(1-p) k^{2} \beta^{\prime} x^{\prime 3}+\frac{1}{8}(1-p)^{2} k^{2} \beta^{\prime} x^{\prime} y^{\prime 2}\right\} \\
& Z_{2}=-2 \mu\left\{(1-p)\left(f_{2}+\bar{f}_{2}\right)+k\left(\zeta f_{3}^{\prime}+\overline{\zeta f_{3}^{\prime}}\right)-\frac{1}{24}(1-p)(3-p) k^{2}{\beta^{\prime} x^{\prime 3}}^{3}\right. \\
& \left.-\frac{1}{8}\left(7+2 p-p^{2}\right) k^{2} \beta^{\prime} x^{\prime} y^{\prime 2}-\frac{1}{2}(3-p)\left(\beta+\beta^{\prime} x^{\prime}\right)\right\} \\
& X_{y^{\prime}}^{\prime}=2 i \mu\left\{f_{1}^{\prime}-\bar{f}_{1}^{\prime}+x^{\prime}\left(f_{2}^{\prime}-\bar{f}_{2}^{\prime}\right)+(1+p)\left(f_{2}-\bar{f}_{2}\right)+\frac{k}{2}\left(\zeta f_{3}^{\prime}-\overline{\zeta f_{3}^{\prime}}\right)\right. \\
& \left.+\frac{i}{8}\left(4+p-p^{2}\right) k^{2} \beta^{\prime} x^{\prime 2} y^{\prime}-\frac{i}{24}\left(6-p-p^{2}\right) k^{2} \beta^{\prime} y^{\prime 3}\right\} \\
& X_{z}^{\prime}=\mu\left\{i\left(f_{3}^{\prime}-\bar{f}_{3}^{\prime}\right)-\alpha y^{\prime}+k \gamma^{\prime} x^{\prime} y^{\prime}\right\}, \\
& Y_{3}^{\prime}=-\mu\left\{f_{3}^{\prime}+\bar{f}_{3}^{\prime}-\alpha x^{\prime}-k \beta^{\prime} y^{\prime 2}+\frac{k \gamma^{\prime}}{2}\left(x^{\prime 2}-y^{\prime 2}\right)\right\} .
\end{aligned}
$$


Take for the bounding curve of the section, the expression

$$
F\left(x^{\prime}, y^{\prime}\right)=0 \text {. }
$$

The conditions for the lateral surface of the rod to be free from external forces are

$$
\left.\begin{array}{r}
X_{x}^{\prime}, \frac{\partial F}{\partial x^{\prime}}+X_{y}^{\prime}, \frac{\partial F}{\partial y^{\prime}}-k D_{2}(F) X_{z}^{\prime}=0 \\
X_{y^{\prime}}^{\prime} \frac{\partial F}{\partial x^{\prime}}+Y_{y^{\prime}}^{\prime} \frac{\partial F}{\partial y^{\prime}}-k D_{2}(F) Y_{z}^{\prime}=0 \\
X_{z}^{\prime} \frac{\partial F}{\partial x^{\prime}}+Y_{z}^{\prime} \frac{\partial F}{\partial y^{\prime}}-\mu k(3-p)\left(\beta+\beta^{\prime} x^{\prime}\right) D_{2}(F)=0 .
\end{array}\right\}
$$

Consider a spiral rod stretched by a pair of axial forces $P$, and imagine a small portion of the rod cut by two parallel planes perpendicular to the axis of rod, as shown in Fig. 1.

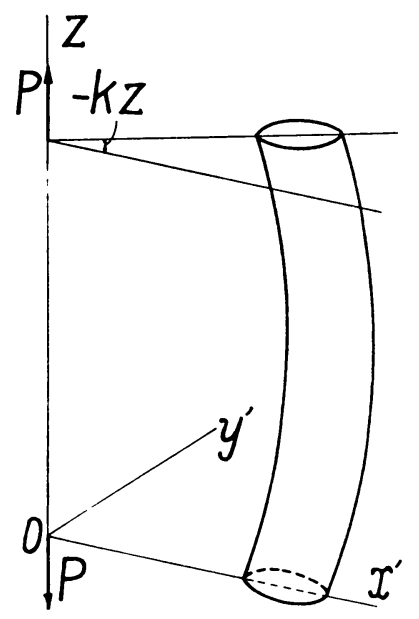

Fig. 1.

The equilibrium condition of the surface tractions for this portion is

$$
\left.\begin{array}{r}
\iint Z_{z} d x^{\prime} d y^{\prime}=P \\
\iint Z_{z} x^{\prime} d x^{\prime} d y^{\prime}=0 \\
\iint\left(y^{\prime} X_{z}^{\prime}-x^{\prime} Y_{z}^{\prime}\right) d x^{\prime} d y^{\prime}=0
\end{array}\right\}
$$

where the integrals are taken over the cross section.

The arbitrary functions $f_{1}, f_{2}$ and $f_{3}$ are determined so as to satisfy the boundary condition (12), and the constants $\alpha, \beta$ and $\beta^{\prime}$ are obtained from the condition (13). 
Substituting the expressions of $X_{z}^{\prime}, Y_{z}^{\prime}$ in (10) into the third Eq. (12), it becomes

$$
\begin{aligned}
\frac{d}{d s}\left(f_{3}+\bar{f}_{3}\right)-\frac{1}{2}\{\alpha+(3-p) k \beta\} & \frac{d}{d s}(\zeta \bar{\zeta}) \\
& -\frac{1}{12}(11-3 p) k \beta^{\prime} \frac{d x^{\prime 3}}{d s}-\frac{1}{4}(5-p) k \beta^{\prime} \frac{d}{d s} x^{\prime} y^{\prime 2}=0,
\end{aligned}
$$

where $d s$ is the element of arc of the bounding curve of the cross section. It follows that the equation

$$
\begin{aligned}
f_{3}+\bar{f}_{3}=\frac{1}{2}\{\alpha+(3-p) k \beta\}\left(x^{\prime 2}+{y^{\prime 2}}^{2}\right. & \\
& +\frac{1}{12}(11-3 p) k \beta^{\prime} x^{\prime 3}+\frac{1}{4}(5-p) k \beta^{\prime} x^{\prime} y^{\prime 2}+\text { const., }
\end{aligned}
$$

holds on the bounding curve, from which $f_{3}$ is determined. For the convenience of further calculations, we shall rewrite the stresses $X_{x}^{\prime}, Y_{v}^{\prime}$ and $X_{v}^{\prime}$ in (11) in the expressions as

$$
\left.\begin{array}{c}
X_{x^{\prime}}^{\prime}=\frac{\partial^{2} x}{\partial y^{\prime \overline{2}}}+2 \mu k\left(\zeta f_{3}^{\prime}+\overline{\zeta f_{3}^{\prime}}\right)+\frac{\mu}{12}(3-p)(1+p) k^{2} \beta^{\prime} x^{\prime 3}-\frac{\mu}{4}\left(9-p^{2}\right) k^{2} \beta^{\prime} x^{\prime}{y^{\prime 2}}^{2} \\
\vdots
\end{array}\right\}
$$

where

$$
\chi=-\varphi_{1}-\bar{\varphi}_{1}-x^{\prime}\left(\varphi_{2}+\bar{\varphi}_{2}\right), \quad \varphi_{1}^{\prime \prime}=2 \mu\left(f_{1}^{\prime}+p f_{2}\right), \quad \varphi_{2}^{\prime}=2 \mu f_{2} .
$$

Substituting these expressions for stresses into the first and second Eqs. (12), by virtue of (14), we obtain

$$
\left.\begin{array}{rl}
\frac{d}{d s}\left(\frac{\partial x}{\partial x^{\prime}}+\right. & \left.i \frac{\partial x}{\partial y^{\prime}}\right)+\mu k \frac{d}{d s}\left[F_{3}(\zeta)-\bar{F}_{3}(\bar{\zeta})\right]+\mu k^{2} \beta^{\prime} \frac{d \Phi}{d s} \\
& -\frac{1}{2}(3-p) \mu k^{2} \beta^{\prime} \zeta \frac{d}{d s}(\bar{\zeta} \bar{\zeta})-\frac{i}{4}(3-p) \mu k^{2} \beta^{\prime} x^{\prime 2} y^{\prime} \frac{d}{d s}(\zeta+3 \bar{\zeta})=0 \\
\frac{d}{d s}\left(\frac{\partial x}{\partial x^{\prime}}-i \frac{\partial x}{\partial y^{\prime}}\right)-\mu k \frac{d}{d s}\left[F_{3}(\zeta)-\bar{F}_{3}(\bar{\zeta})\right]+\mu k^{2} \beta^{\prime} \frac{d \Phi}{d s} \\
-\frac{1}{2}(3-p) \mu k^{2} \beta^{\prime} \bar{\zeta} \frac{d}{d s}(\zeta \bar{\zeta})+\frac{i}{4}(3-p) \mu k^{2} \beta^{\prime} x^{\prime 2} y^{\prime} \frac{d}{d s}(3 \zeta+\bar{\zeta})=0,
\end{array}\right\}
$$

where

$$
F_{3}^{\prime}=2 \zeta f_{3}^{\prime}
$$


$\Phi=-\frac{1}{48}\left[(11+p)(3-p) x^{4}+6(1-p)^{2} x^{\prime 2} y^{\prime 2}-\left(9-2 p+p^{2}\right){y^{\prime}}^{4}\right]$

$$
+\frac{i}{12}(3-p)\left[(1+p){x^{\prime 2}}^{2}(7+p){y^{\prime 2}}^{2}\right] x^{\prime} y^{\prime}
$$

From (16), $f_{1}$ and $f_{2}$ are obtained.

As an example of the procedure, consider an elliptic spiral rod whose cross section is

$$
\left(x^{\prime}-c\right)^{2} / a^{2}+y^{\prime 2} / b^{2}=1,
$$

as shown in Fig. 2.

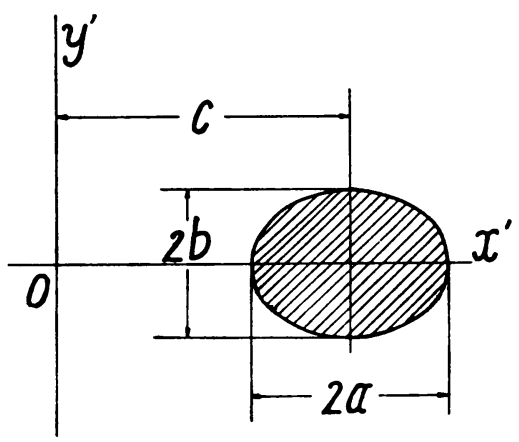

Frg. 2.

Transform now the elliptic section in the $\zeta$-plane into a unit circle in the $t$-plane by the equation

$$
\zeta=c+a^{\prime} t+b^{\prime} t^{-1}
$$

where $a^{\prime}+b^{\prime}=a, a^{\prime}-b^{\prime}=b$.

Take for the function $f_{3}$, the expression

$$
f_{3}=c_{1}\left(t+s t^{-1}\right)+c_{2}\left(t^{2}+s^{2} t^{-2}\right)+c_{3}\left(t^{3}+s^{3} t^{-3}\right),
$$

where $S=b^{\prime} / a^{\prime}$. The unknown coefficients $c_{1}, c_{2}$ and $c_{3}$ are readily obtained from the boundary condition (14).

Remembering the condition that $\varphi_{1}$ and $\varphi_{2}$ are analytic at any point of the section in the $t$-plane, we take for the functions the expressions

$$
\varphi_{1}^{\prime}=\sum_{n=1}^{4} A_{n}\left(t^{n}+s^{n} t^{-n}\right), \quad \varphi_{2}=\sum_{n=1}^{4} B_{n}\left(t^{n}+s^{n} t^{-n}\right),
$$

where $A_{n}$ and $B_{n}$ are real constants. These unknown constants are obtained from the boundary condition (16), and the other unknown constants $\alpha, \beta$ and $\beta^{\prime}$ are finally obtained from the conditions (13).

In the case of stretching, the predominating stress is the normal stress $Z_{2}$ and the shearing stresses $X_{z}^{\prime}$ and $Y_{z}^{\prime}$ follow it, but the latter are smaller quantities of the order $k$. The other stresses are of the order $k^{2}$ and are very small quantities when $k$ is small. When the section of the spiral rod is a circle of radius unity, then $a=b=1$, and it 
follows $a^{\prime}=1, b^{\prime}=0, s=0$. The main stress $Z_{z}$ for the circular section, referred to the polar coordinates with the pole at the center of the circle, is

$Z_{s}=\mu(3-p)\left(\beta+\beta^{\prime} c\right)-2(1-p) B_{1}-4 \mu k c_{1} c$

$$
\begin{aligned}
& +\left[\mu \beta^{\prime}(3-p)-4(1-p) B_{2}-4 \mu k\left(2 c_{2} c+c_{1}\right)\right] r \cos \theta \\
& -2\left[3(1-p) B_{3}+2 \mu k\left(3 c_{3} c+2 c_{2}\right)\right] r^{2} \cos 2 \theta-4\left[2(1-p) B_{4}+3 \mu k c_{3}\right] r^{3} \cos 3 \theta \\
& +\frac{1}{12} \mu k^{2} \beta^{\prime}(1-p)(3-p)(c+r \cos \theta)^{3} \\
& +\frac{1}{4} \mu k^{2} \beta^{\prime}\left(7+2 p-p^{2}\right)(c+r \cos \theta) r^{2} \sin ^{2} \theta .
\end{aligned}
$$

From the third Eq. (13), we have

$$
\alpha=\frac{1}{2}(1-p) k \beta^{\prime} c .
$$

Hence, a twist almost proportional to $k$ arises when a spiral rod with a circular section

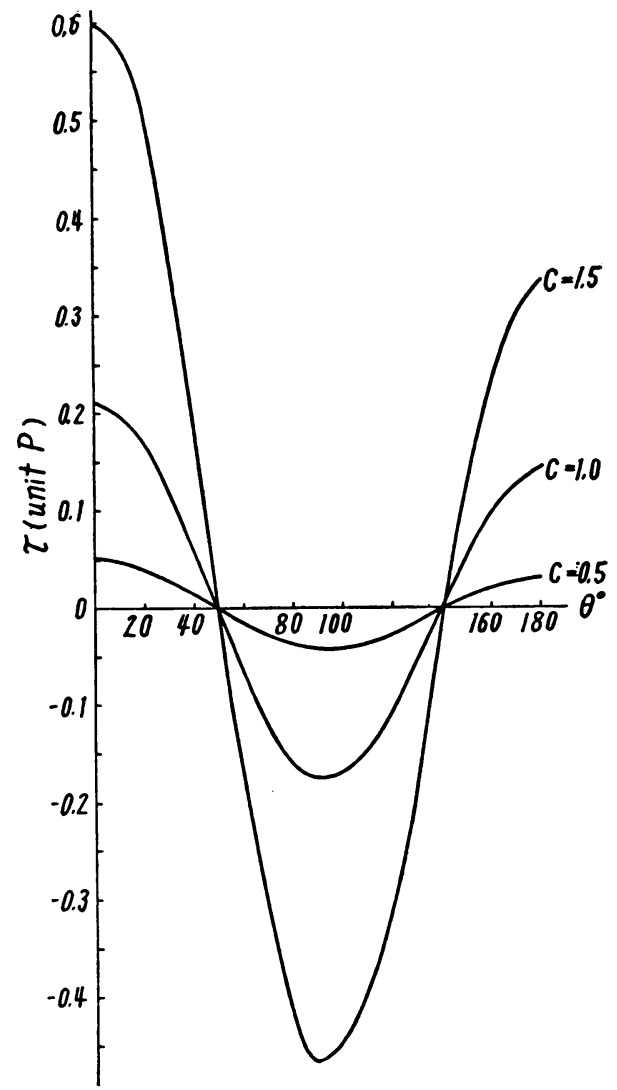

Fig. 3. The shearing stress along the bounding circle, when $k=0.25$. 
is pulled axially. ${ }^{2}$ From the remaining Eqs. (13), the other unknown constants $\beta$ and $\beta^{\prime}$ are obtained.

If $\tau$ be the shearing stress along the bounding circle of the section, it becomes

\}$=-\mu\left\{\left[2 c_{1}-\frac{1}{4} k \beta^{\prime} c^{2}(1-p)-\frac{1}{4} p k \beta^{\prime}\right] \cos \theta+4 c_{2} \cos 2 \theta+\left(6 c_{3}+\frac{1}{4} k \beta^{\prime}\right) \cos 3 \theta\right\}$.

The shearing stress along the periphery has been calculated from (23) for various values of $c$, assuming the Poisson's ratio and $k$ to be 0.3 and 0.25 , respectively, and is shomn in Fig. 3. As is seen from the figure, the shearing stress becomes large at both ends of two diameters parallel to the coordinate axes $\left(x^{\prime}, y^{\prime}\right)$, and attains its maximum value at the outer end of the diameter on the $x^{\prime}$-axis. The distribution of the normal tension $Z$, on the axis of $x^{\prime}$, obtained from (21), is given in Fig. 4. For the sake of comparison, the

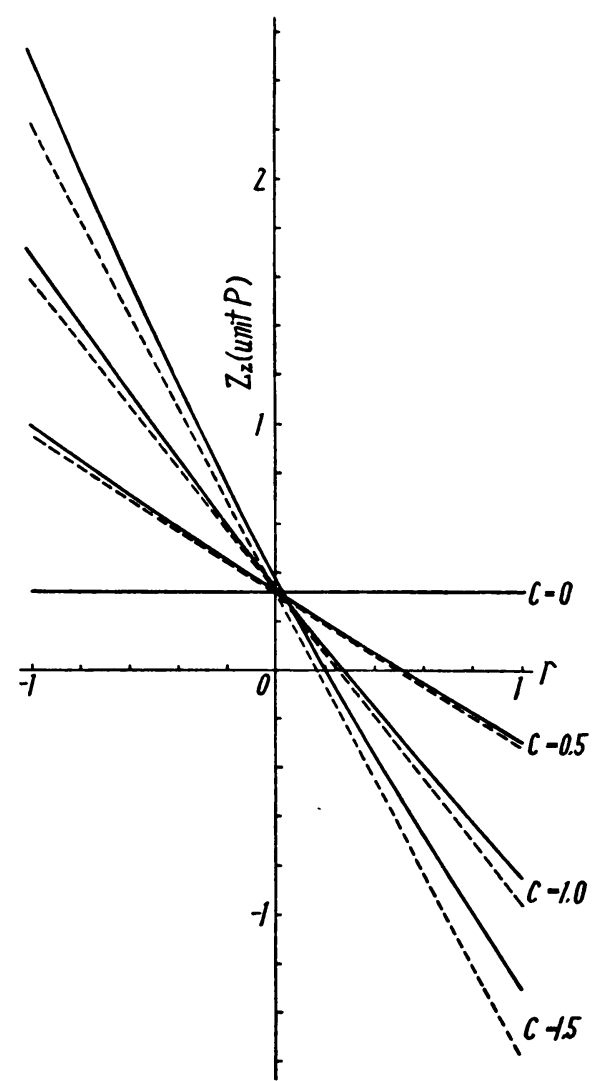

FIG. 4. The distribution of $Z$, on the $x^{\prime}$-axis, when $k=0.25$.

corresponding distribution of $Z$, for a straight rod is also shown by dotted lines in the same figure.

Acknowledgment. A grant for science research has been given for this study by the Education Ministry of Japan.

${ }^{2} \beta^{\prime}$ is a function of $k$ and $c$, but it remains almost constant for the variation of $k$, when $k$ is small. 